\title{
The chord length distribution function of a non-convex hexagon
}

\author{
Uwe Bäsel $^{1 *}$, Vittoria Bonanzinga ${ }^{2}$, Andrei Duma ${ }^{3}$ \\ ${ }^{1}$ HTWK Leipzig, University of Applied Sciences, Faculty of Mechanical and \\ Energy Engineering, Germany \\ ${ }^{2}$ Università degli Studi di Reggio Calabria, Dipartimento di Ingegneria \\ dell'Informazione, delle Infrastrutture e dell'Energia Sostenibile, Italy \\ ${ }^{3}$ FernUniversität in Hagen, Fakultät für Mathematik und Informatik, Germany \\ *Email address for correspondence: uwe.baesel@htwk-leipzig.de \\ Communicated by Mario Primicerio \\ Received on 12 19, 2016. Accepted on 12 20, 2017.
}

\begin{abstract}
In this paper we obtain the chord length distribution function of a non-convex equilateral hexagon and then derive the associated density function. Finally, we calculate the expected value of the chord length.

Keywords: Non-convex hexagon, chord length distribution function, multiple chord distribution, chord power integrals

AMS subject classification: 60D05, 52A22
\end{abstract}

\section{Introduction}

On the one hand, chord length distributions of plane figures and spatial bodies are studied for theoretical reasons (see [17], [12], [7]), on the other hand, there are numerous practical applications such as in acoustics, ecology, image analysis, stereology, and reactor design (see [14, p. 6195] for further bibliographical references). An important application one finds in the small-angle scattering (SAS) for the investigation of material structures using neutron beams or X-rays in order to get information about the average size and the size distribution of particles [8], [9].

The chord length distribution functions for a number of planar convex figures are already known. Please refer to the following results:

- regular hexagon [4],

- arbitrary triangles [5], [3],

- rectangular trapezium [6],

- isosceles trapezium [16], 


\section{The chord length distribution function of a non-convex hexagon}

- every regular polygon [11], [2], where in [2] also the distribution function is determined for the distance between two random points uniformly distributed in a regular polygon.

There are straight lines that cross a non-convex body more than one time. So for non-convex bodies we have to distinguish between the chord length distribution function of the one chord distribution (OCD-function) and those of the multiple chord distribution (MCD-function) [14, p. 6195]. If the intersection $S=G \cap \mathcal{F}$ of a random straight line $G$ and a non-convex body $\mathcal{F}$ consists of several line segments $S_{i}, i=1,2, \ldots, n$, every $S_{i}$ has to be counted as one chord of length $\left|S_{i}\right|$ in order to obtain the MCD-function. In the case of the OCD-function, all line segments of $S$ are considered as one chord of length $|S|=\sum_{i=1}^{n}\left|S_{i}\right|$.

The OCD-function and the MCD-function for a non-convex polygon (Kshaped pentagon) have been determined in [1], the MCD-function by means of simulation and analytical computation, the OCD-function by means of simulation.

In the present paper the notion chord length distribution function always means MCD-function. We determine this function and the associated density function for the non-convex equilateral hexagon $\mathcal{H}$ (see Fig. 1) in Section 2, and draw some further conclusions in Section 3.

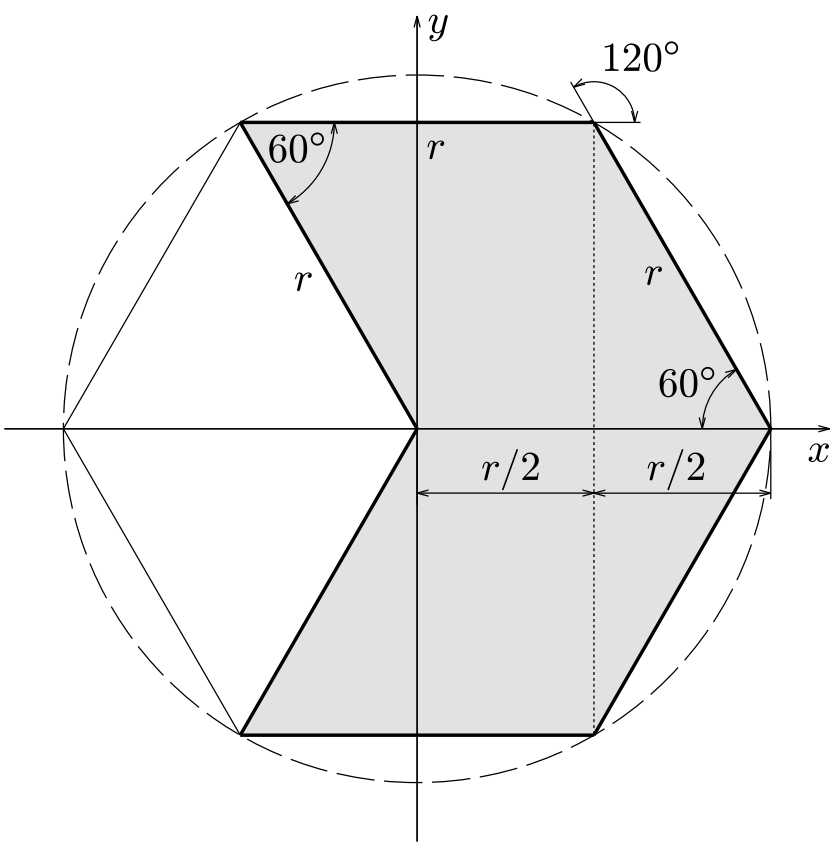

Figure 1. The non-convex equilateral hexagon $\mathcal{H}$ (grey-shaded) 


\section{U. Bäsel, V. Bonanzinga, A. Duma}

\section{The distribution function}

The measure $m$ for the set $\mathcal{S}$ of all chords of $\mathcal{H}$ is defined by

$$
m(\mathcal{S})=\int_{G \cap \mathcal{H} \neq \emptyset} \#\left(S_{i}: S_{i} \subset G\right) \mathrm{d} G=\int_{0}^{2 \pi} \int_{\mathbb{R}^{+}} \#\left(S_{i}: S_{i} \subset G\right) \mathrm{d} p \mathrm{~d} \varphi
$$

(see [1, p. 142]), where $\mathrm{d} G=\mathrm{d} p \mathrm{~d} \varphi$ is the density for sets of straight lines, $\varphi$ is the angle of the normal of the line $G$ with the $x$ axis, and $p$ is the distance of $G$ from the origin. So the equation of $G$ is given by

$$
x \cos \varphi+y \sin \varphi-p=0 .
$$

Up to a constant factor, $\mathrm{d} G$ is the only density that is invariant under planar motions $[15$, p. 28]. Considering $p$ as the signed distance from the origin, we may restrict $\varphi$ to the interval $0 \leq \varphi \leq \pi$ and get

$$
m(\mathcal{S})=\int_{0}^{\pi} \int_{\mathbb{R}} \#\left(S_{i}: S_{i} \subset G\right) \mathrm{d} p \mathrm{~d} \varphi .
$$

For the calculation of $m(\mathcal{S})$ we need the width $w(\varphi)$ of $\mathcal{H}$ in the direction $\varphi$. This width function is, as easily verifiable, given by

$$
w(\varphi)=\left\{\begin{array}{cl}
w_{1}(\varphi), & 0 \leq \varphi<\pi / 6, \\
w_{2}(\varphi), & \pi / 6 \leq \varphi<\pi / 2 \\
-w_{2}(-\varphi), & \pi / 2 \leq \varphi<5 \pi / 6, \\
-w_{1}(-\varphi), & 5 \pi / 6 \leq \varphi \leq \pi,
\end{array}\right.
$$

where

$$
w_{1}(\varphi)=\sqrt{3} r \cos \left(\frac{\pi}{6}-\varphi\right) \quad \text { and } \quad w_{2}(\varphi)=2 r \cos \left(\frac{\pi}{3}-\varphi\right) .
$$

We note that $w(\varphi)$ is symmetrical with respect to the line $\varphi=\pi / 2$. For $0 \leq \varphi \leq \pi / 6$ the lines in the strip of the width

$$
t_{2}(\varphi)=r \sin \left(\frac{\pi}{6}-\varphi\right)
$$

(see Fig. 4) have to be counted twice, since each of these lines carries two chords. Taking into account the symmetry of $\mathcal{H}$, we get

$$
\begin{aligned}
m(\mathcal{S}) & =2 \int_{0}^{\pi / 2} \int_{\mathbb{R}} \#\left(S_{i}: S_{i} \subset G\right) \mathrm{d} p \mathrm{~d} \varphi \\
& =2\left\{\int_{0}^{\pi / 2} w(\varphi) \mathrm{d} \varphi+\int_{0}^{\pi / 6} t_{2}(\varphi) \mathrm{d} \varphi\right\}
\end{aligned}
$$




$$
=2\left\{\left(2 r+\frac{\sqrt{3}}{2} r\right)+\left(r-\frac{\sqrt{3}}{2} r\right)\right\}=6 r .
$$

Alternatively, this result immediately follows from the measure for the set of all lines that intersect a regular hexagon. For every bounded convex set, this measure is equal to its perimeter (see e. g. [15, p. 30]), hence for the regular hexagon of side length $r$, it is equal to $6 r$. The difference between the measures for the sets of lines intersecting the regular hexagon and the non-convex hexagon $\mathcal{H}$ is equal to

$$
2 \int_{0}^{\pi / 6} t_{2}(\varphi) \mathrm{d} \varphi
$$

But this is also the measure for the set of all lines $G$ intersecting $\mathcal{H}$ with $\#\left(S_{i}: S_{i} \subset G\right)=2$.

The measure for all chords $S^{\prime} \in \mathcal{S}$ of length $\left|S^{\prime}\right| \leq s$ is given by

$$
\begin{aligned}
m\left(S^{\prime}:\left|S^{\prime}\right| \leq s\right) & =\int_{G \cap \mathcal{H} \neq \emptyset} \#\left(S_{i}: S_{i} \subset G,\left|S_{i}\right| \leq s\right) \mathrm{d} G \\
& =\int_{0}^{\pi} \int_{\mathbb{R}} \#\left(S_{i}: S_{i} \subset G,\left|S_{i}\right| \leq s\right) \mathrm{d} p \mathrm{~d} \varphi .
\end{aligned}
$$

It follows that the distribution function $F$ for the length of a random chord $S^{\prime}$ of $\mathcal{H}$ is defined by

$$
F(s)=P\left(\left|S^{\prime}\right| \leq s\right)=\frac{m\left(S^{\prime}:\left|S^{\prime}\right| \leq s\right)}{m(\mathcal{S})}
$$

(see [1], Eq. (2)). In the present case, therefore

$$
F(s)=\frac{1}{6 r} m\left(S^{\prime}:\left|S^{\prime}\right| \leq s\right) .
$$

Concerning the chord length $s$, it is necessary to distinguish between the four cases:
1) $0 \leq s \leq \frac{\sqrt{3}}{2} r$
2) $\frac{\sqrt{3}}{2} r \leq s \leq r$
3) $r \leq s \leq \sqrt{3} r$
4) $\sqrt{3} r \leq s \leq 2 r$.

In the following, the values

$$
\varphi_{0}(s)=\arccos \frac{\sqrt{3} r}{2 s}, \quad \varphi_{1}(s)=\arcsin \frac{\sqrt{3} r}{2 s}, \quad \varphi_{2}(s)=\arcsin \frac{\sqrt{3} r}{s}
$$

of the angle $\varphi$ are needed for the integration limits. 


\section{U. Bäsel, V. Bonanzinga, A. Duma}

Now we consider Case 1:

a) For fixed value of the angle $\varphi \in[0, \pi / 6]$, the set of chords with length $\leq s$ consists of three strips (see Fig. 2) with the widths

$$
\begin{aligned}
& h_{1}(s, \varphi)=\frac{s}{\sqrt{3}}\left[\frac{1}{2}+\sin \left(2 \varphi+\frac{\pi}{6}\right)\right], h_{2}(s, \varphi)=\frac{s}{\sqrt{3}}\left[\frac{1}{2}-\sin \left(2 \varphi-\frac{\pi}{6}\right)\right], \\
& h_{3}(s, \varphi)=\frac{s}{\sqrt{3}}\left(\cos 2 \varphi-\frac{1}{2}\right) .
\end{aligned}
$$

We note that the first two strips partially overlap if the respective lines carry two chords.

b) For $\varphi \in[\pi / 6, \pi / 2]$ the set of chords with length $\leq s$ consists of two non-overlapping strips (Fig. 3) of widths $h_{1}(s, \varphi)$ (see a) and

$$
h_{4}(s, \varphi)=\frac{s}{\sqrt{3}}\left[\cos \left(2 \varphi-\frac{2 \pi}{3}\right)-\frac{1}{2}\right] .
$$

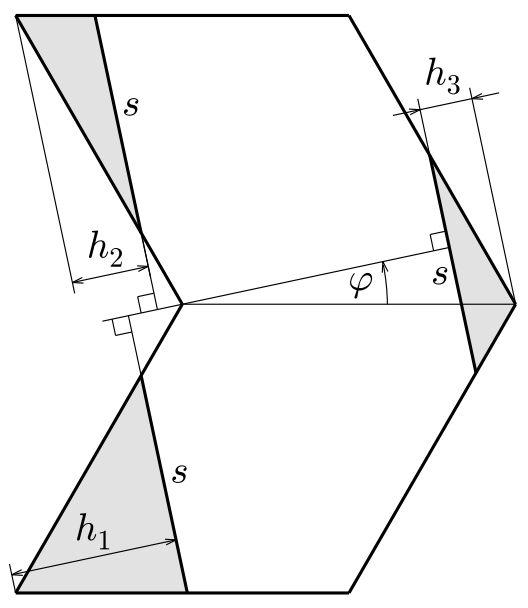

Figure 2. Case 1a

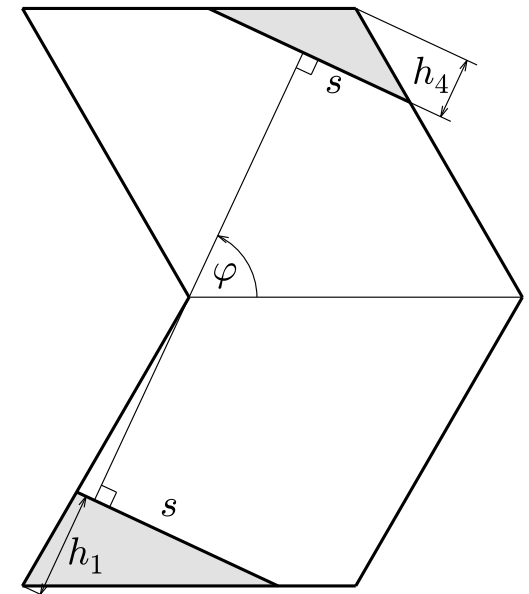

Figure 3. Case 1b

Therefore, the restriction $F_{1}(s)$ of the chord length distribution function $F(s)$ for Case 1 is given by

$$
\begin{aligned}
F_{1}(s)= & \frac{1}{3 r}\left\{\int_{0}^{\pi / 6}\left[h_{1}(s, \varphi)+h_{2}(s, \varphi)+h_{3}(s, \varphi)\right] \mathrm{d} \varphi\right. \\
& \left.+\int_{\pi / 6}^{\pi / 2}\left[h_{1}(s, \varphi)+h_{4}(s, \varphi)\right] \mathrm{d} \varphi\right\}=\frac{15 \sqrt{3}+\pi}{36 \sqrt{3}} \frac{s}{r} .
\end{aligned}
$$




\section{The chord length distribution function of a non-convex hexagon}

Now we consider Case 2:

a) $\varphi \in\left[0, \varphi_{0}(s)\right]$ (Fig. 4$)$ : The set of chords with length $\leq s$ consists of three strips of widths

$$
t_{1}(\varphi)=r \sin \left(\varphi+\frac{\pi}{6}\right), \quad t_{2}(\varphi)=r \sin \left(\frac{\pi}{6}-\varphi\right),
$$

and (see Case 1a) $h_{3}(s, \varphi)$.

b) $\varphi \in\left[\varphi_{0}(s), \pi / 6\right]$ : The set of chords with length $\leq s$ consists of three strips of widths $h_{1}(s, \varphi), h_{2}(s, \varphi)$ and $h_{3}(s, \varphi)$ (see Case 1a).

c) $\varphi \in\left[\pi / 6, \varphi_{1}(s)-\pi / 6\right]$ : There are two strips of widths $h_{1}(s, \varphi)$ and $h_{4}(s, \varphi)$ (see Case $\left.1 \mathrm{~b}\right)$.

d) $\varphi \in\left[\varphi_{1}(s)-\pi / 6,5 \pi / 6-\varphi_{1}(s)\right]$ (Fig. 5): One gets

$$
\ell_{1}(s, \varphi)=\frac{s}{\sqrt{3}}\left(\frac{1}{2}-\cos 2 \varphi\right), \quad \ell_{2}(\varphi)=r \cos \varphi
$$

and (see Case 1b) $h_{4}(s, \varphi)$.

e) $\varphi \in\left[5 \pi / 6-\varphi_{1}(s), \pi / 2\right]$ : Here we have two strips of widths $h_{1}(s, \varphi)$ and $h_{4}(s, \varphi)$ (see Case 1b).

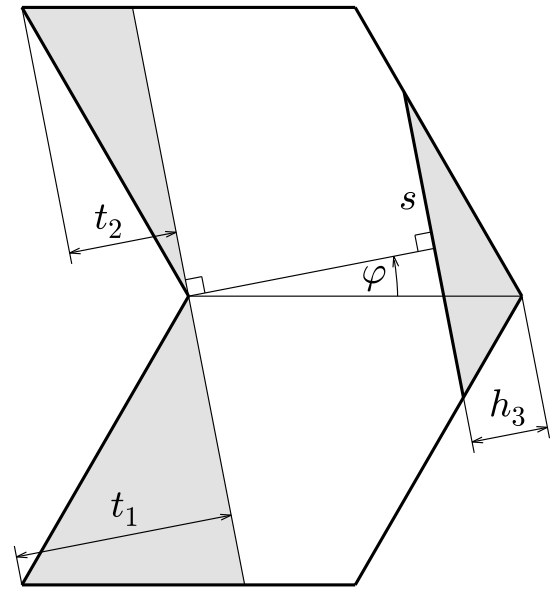

Figure 4. Case 2a

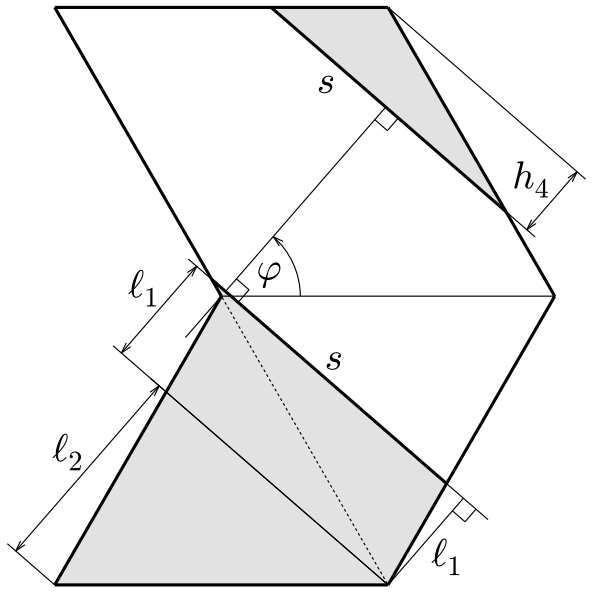

Figure 5. Case 2d

Hence the restriction $F_{2}$ of $F$ for Case 2 is given by

$$
\begin{aligned}
F_{2}(s)= & \frac{1}{3 r}\left\{\int_{0}^{\varphi_{0}(s)}\left[t_{1}(\varphi)+t_{2}(\varphi)+h_{3}(s, \varphi)\right] \mathrm{d} \varphi\right. \\
& +\int_{\varphi_{0}(s)}^{\pi / 6}\left[h_{1}(s, \varphi)+h_{2}(s, \varphi)+h_{3}(s, \varphi)\right] \mathrm{d} \varphi
\end{aligned}
$$




\section{U. Bäsel, V. Bonanzinga, A. Duma}

$$
\begin{aligned}
& +\int_{\pi / 6}^{\varphi_{1}(s)-\pi / 6}\left[h_{1}(s, \varphi)+h_{4}(s, \varphi)\right] \mathrm{d} \varphi \\
& +\int_{\varphi_{1}(s)-\pi / 6}^{5 \pi / 6-\varphi_{1}(s)}\left[\ell_{1}(s, \varphi)+\ell_{2}(\varphi)+h_{4}(s, \varphi)\right] \mathrm{d} \varphi \\
& \left.+\int_{5 \pi / 6-\varphi_{1}(s)}^{\pi / 2}\left[h_{1}(s, \varphi)+h_{4}(s, \varphi)\right] \mathrm{d} \varphi\right\} \\
& =\frac{15 \sqrt{3}+\pi-12 \varphi_{0}(s)}{36 \sqrt{3}} \frac{s}{r}+\frac{1}{4} \sqrt{4-3\left(\frac{r}{s}\right)^{2}} .
\end{aligned}
$$

We consider Case 3:

a) $\varphi \in\left[0, \varphi_{1}(s)-\pi / 6\right]: t_{1}(\varphi), t_{2}(\varphi)$ and $h_{3}(s, \varphi)$ (see Case 2a).

b) $\varphi \in\left[\varphi_{1}(s)-\pi / 6, \pi / 6\right]$ (Fig. 6): $t_{1}(\varphi), t_{2}(\varphi)$ (see Case 2a) and

$$
u_{1}(\varphi)=r \sin \left(\frac{\pi}{6}-\varphi\right), \quad u_{2}(s, \varphi)=\frac{s}{\sqrt{3}}\left[\frac{1}{2}+\sin \left(2 \varphi+\frac{\pi}{6}\right)\right]-r \cos \varphi
$$

c) $\varphi \in\left[\pi / 6, \pi / 2-\varphi_{1}(s)\right]$ (Fig. 7): $\ell_{1}(s, \varphi), \ell_{2}(\varphi)$ (Case 2d) and $u_{2}(s, \varphi)$ (Case 3b).

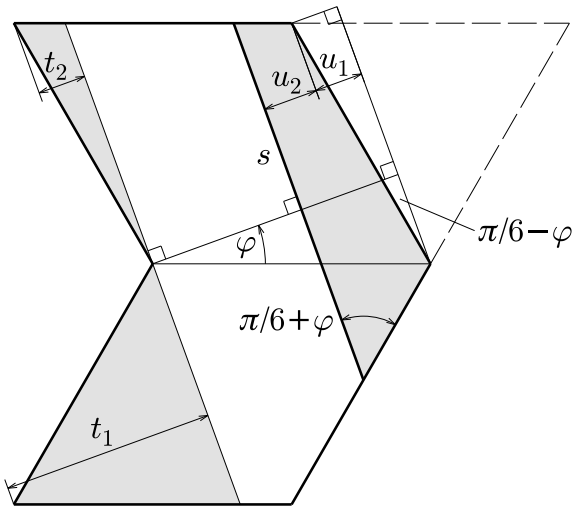

Figure 6. Case 3b

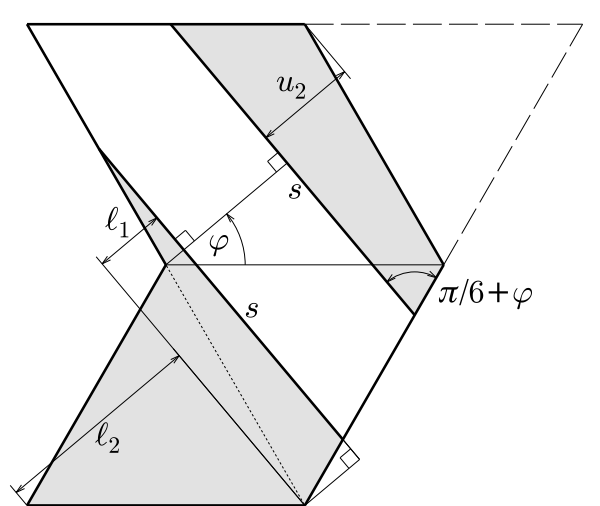

Figure 7. Case 3c

d) $\varphi \in\left[\pi / 2-\varphi_{1}(s), \pi / 6+\varphi_{1}(s)\right]: \ell_{1}(s, \varphi), \ell_{2}(\varphi)$ and $h_{4}(s, \varphi)$ (see Case $2 \mathrm{~d})$.

e) $\varphi \in\left[\pi / 6+\varphi_{1}(s), \pi / 2\right]$ (Fig. 8): The lengths of all chords in the direction perpendicular to $\varphi$ are $\leq s$, and there are no lines that carry two chords. Hence the width of the strip is equal to the width function $w_{2}(\varphi)$. 


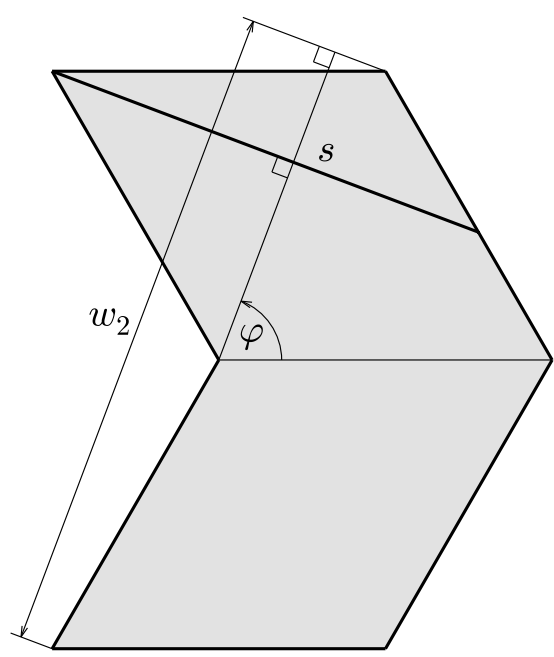

Figure 8. Case 3e

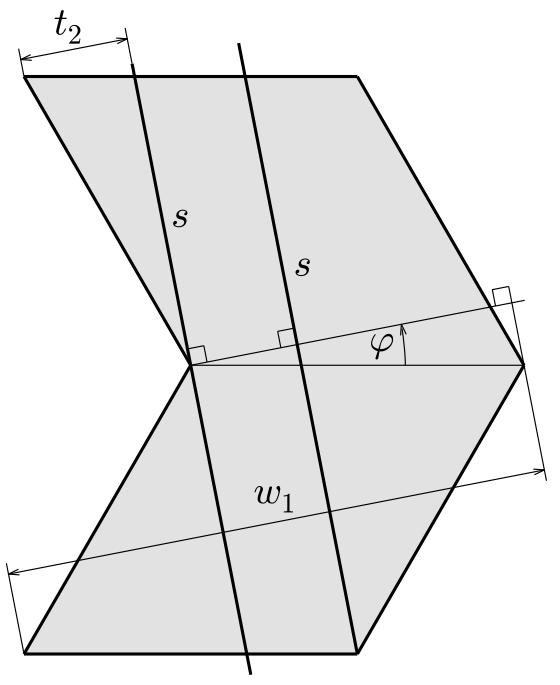

Figure 9. Case 4a

So we have

$$
\begin{aligned}
F_{3}(s)= & \frac{1}{3 r}\left\{\int_{0}^{\varphi_{1}(s)-\pi / 6}\left[t_{1}(\varphi)+t_{2}(\varphi)+h_{3}(s, \varphi)\right] \mathrm{d} \varphi\right. \\
& +\int_{\varphi_{1}(s)-\pi / 6}^{\pi / 6}\left[t_{1}(\varphi)+t_{2}(\varphi)+u_{1}(\varphi)+u_{2}(s, \varphi)\right] \mathrm{d} \varphi \\
& +\int_{\pi / 6}^{\pi / 2-\varphi_{1}(s)}\left[\ell_{1}(s, \varphi)+\ell_{2}(\varphi)+u_{2}(s, \varphi)\right] \mathrm{d} \varphi \\
& \left.+\int_{\pi / 2-\varphi_{1}(s)}^{\pi / 6+\varphi_{1}(s)}\left[\ell_{1}(s, \varphi)+\ell_{2}(\varphi)+h_{4}(s, \varphi)\right] \mathrm{d} \varphi+\int_{\pi / 6+\varphi_{1}(s)}^{\pi / 2} w_{2}(\varphi) \mathrm{d} \varphi\right\} \\
= & \frac{2}{3}-\frac{3 \sqrt{3}-7 \pi+24 \varphi_{1}(s)}{36 \sqrt{3}} \frac{s}{r}+\frac{1}{12} \sqrt{4-3\left(\frac{r}{s}\right)^{2}} .
\end{aligned}
$$

\section{Case 4:}

a) $\varphi \in\left[0, \pi / 2-\varphi_{2}(s)\right]$ (Fig. 9): The lengths of all chords in the direction perpendicular to $\varphi$ are $\leq s$. The lines in the strip of width of $t_{2}(\varphi)$ carry two chords. Therefore, $w_{1}(\varphi)+t_{2}(\varphi)$ is the measure for all chords with length $\leq s$ for fixed angle $\varphi$.

b) $\varphi \in\left[\pi / 2-\varphi_{2}(s), \pi / 6\right]: t_{1}(\varphi), t_{2}(\varphi), u_{1}(\varphi), u_{2}(s, \varphi)$ (see Case $3 \mathrm{~b}$ ).

c) $\varphi \in\left[\pi / 6, \varphi_{2}(s)-\pi / 6\right]: \ell_{1}(s, \varphi), \ell_{2}(\varphi), u_{2}(s, \varphi)$ (see Case 3c).

d) $\varphi \in\left[\varphi_{2}(s)-\pi / 6, \pi / 2\right]: w_{2}(\varphi)$ (see Case $3 \mathrm{e}$ ). 


\section{U. Bäsel, V. Bonanzinga, A. Duma}

We get

$$
\begin{aligned}
F_{4}(s)= & \frac{1}{3 r}\left\{\int_{0}^{\pi / 2-\varphi_{2}(s)}\left[w_{1}(\varphi)+t_{2}(\varphi)\right] \mathrm{d} \varphi\right. \\
& +\int_{\pi / 2-\varphi_{2}(s)}^{\pi / 6}\left[t_{1}(\varphi)+t_{2}(\varphi)+u_{1}(\varphi)+u_{2}(s, \varphi)\right] \mathrm{d} \varphi \\
& \left.+\int_{\pi / 6}^{\varphi_{2}(s)-\pi / 6}\left[\ell_{1}(\varphi)+\ell_{2}(\varphi)+u_{2}(s, \varphi)\right] \mathrm{d} \varphi+\int_{\varphi_{2}(s)-\pi / 6}^{\pi / 2} w_{2}(\varphi) \mathrm{d} \varphi\right\} \\
= & \frac{2}{3}-\frac{\pi-3 \varphi_{2}(s)}{6 \sqrt{3}} \frac{s}{r}+\frac{2}{3} \sqrt{1-3\left(\frac{r}{s}\right)^{2}} .
\end{aligned}
$$

By simplifying the previous results, one finds the following theorem.

Theorem 2.1. The distribution function $F$ of the chord length $s$ of the non-convex hexagon $\mathcal{H}$ is given by

$$
F(s)= \begin{cases}0 & \text { if }-\infty<s<0, \\ \left(15+\frac{\pi}{\sqrt{3}}\right) \frac{s}{36 r} & \text { if } 0 \leq s<h, \\ \left(3-\frac{\pi}{\sqrt{3}}\right) \frac{5 s}{36 r}+\frac{s \psi(h / s)}{3 \sqrt{3} r}+\frac{R(h / s)}{2} & \text { if } h \leq s<r, \\ \frac{2}{3}-\left(3-\frac{7 \pi}{\sqrt{3}}\right) \frac{s}{36 r}-\frac{2 s \psi(h / s)}{3 \sqrt{3} r}+\frac{R(h / s)}{6} & \text { if } \quad r \leq s<2 h, \\ \frac{2}{3}-\frac{\pi s}{6 \sqrt{3} r}+\frac{s \psi(2 h / s)}{2 \sqrt{3} r}+\frac{2 R(2 h / s)}{3} & \text { if } 2 h \leq s<2 r, \\ 1 & \text { if } 2 r \leq s<\infty,\end{cases}
$$

where

$$
h=\frac{\sqrt{3} r}{2}, \quad \psi(x)=\arcsin x, \quad R(x)=\sqrt{1-x^{2}} .
$$

The graph of $F$ is shown in Fig. 10 (solid line). As a comparison we show the distribution function of the regular hexagon of side length $r$ (dashed line) according to [10] and [2, Theorem 1]. With

$$
\begin{aligned}
\frac{\mathrm{d} \psi(x)}{\mathrm{d} x} & =\frac{1}{\sqrt{1-x^{2}}}, \quad \frac{\mathrm{d} R(x)}{\mathrm{d} x}=-\frac{x}{\sqrt{1-x^{2}}}, \\
x & =\chi(s)=\frac{a}{s}, \quad \frac{\mathrm{d} \chi(s)}{\mathrm{d} s}=-\frac{a}{s^{2}},
\end{aligned}
$$




\section{The chord length distribution function of a non-convex hexagon}

we easily get the density function $f$ of the chord length $s$ :

$$
f(s)=\left\{\begin{array}{lll}
0 & \text { if } \quad-\infty<s<0 \\
f_{1}(s) & \text { if } \quad 0 \leq s<h \\
f_{2}(s) & \text { if } \quad h \leq s<r \\
f_{3}(s) & \text { if } \quad r \leq s<2 h \\
f_{4}(s) & \text { if } \quad 2 h \leq s<2 r \\
0 & \text { if } \quad 2 r \leq s<\infty
\end{array}\right.
$$

where

$$
\begin{aligned}
& f_{1}(s)=\left(15+\frac{\pi}{\sqrt{3}}\right) \frac{1}{36 r}, \\
& f_{2}(s)=\left(3-\frac{\pi}{\sqrt{3}}\right) \frac{5}{36 r}+\frac{1}{3 \sqrt{3} r}\left(\psi(h / s)-\frac{h}{s R(h / s)}\right)+\frac{h^{2}}{2 s^{3} R(h / s)}, \\
& f_{3}(s)=-\left(3-\frac{7 \pi}{\sqrt{3}}\right) \frac{1}{36 r}-\frac{2}{3 \sqrt{3} r}\left(\psi(h / s)-\frac{h}{s R(h / s)}\right)+\frac{h^{2}}{6 s^{3} R(h / s)}, \\
& f_{4}(s)=-\frac{\pi}{6 \sqrt{3} r}+\frac{1}{2 \sqrt{3} r}\left(\psi(2 h / s)-\frac{2 h}{s R(2 h / s)}\right)+\frac{8 h^{2}}{3 s^{3} R(2 h / s)} .
\end{aligned}
$$

For the right-hand limits at the two discontinuities of $f$ (see Fig. 11), with the abbreviation $h=\sqrt{3} r / 2$ we find

$$
\lim _{s \rightarrow h+0} f(s)=\lim _{s \rightarrow h} f_{2}(s)=\infty, \quad \lim _{s \rightarrow 2 h+0} f(s)=\lim _{s \rightarrow 2 h} f_{4}(s)=\infty .
$$

Furthermore, we have

$$
\lim _{s \rightarrow 2 h-0} f(s)=\lim _{s \rightarrow 2 h-0} f_{3}(s)=\frac{6+\sqrt{3} \pi}{36 r} \approx \frac{0.317817}{r} .
$$




\section{U. Bäsel, V. Bonanzinga, A. Duma}

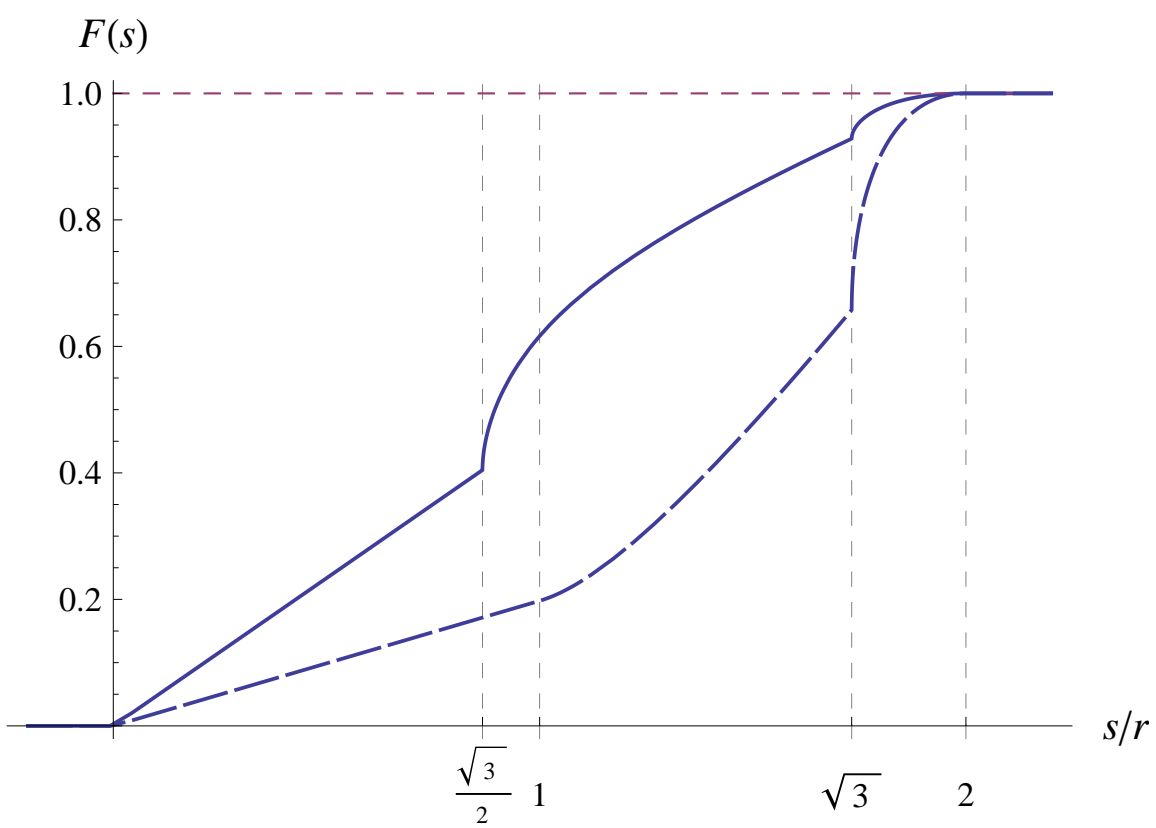

Figure 10. Chord length distribution functions of $\mathcal{H}$ (solid line) and of the regular hexagon of side length $r$ (dashed line)

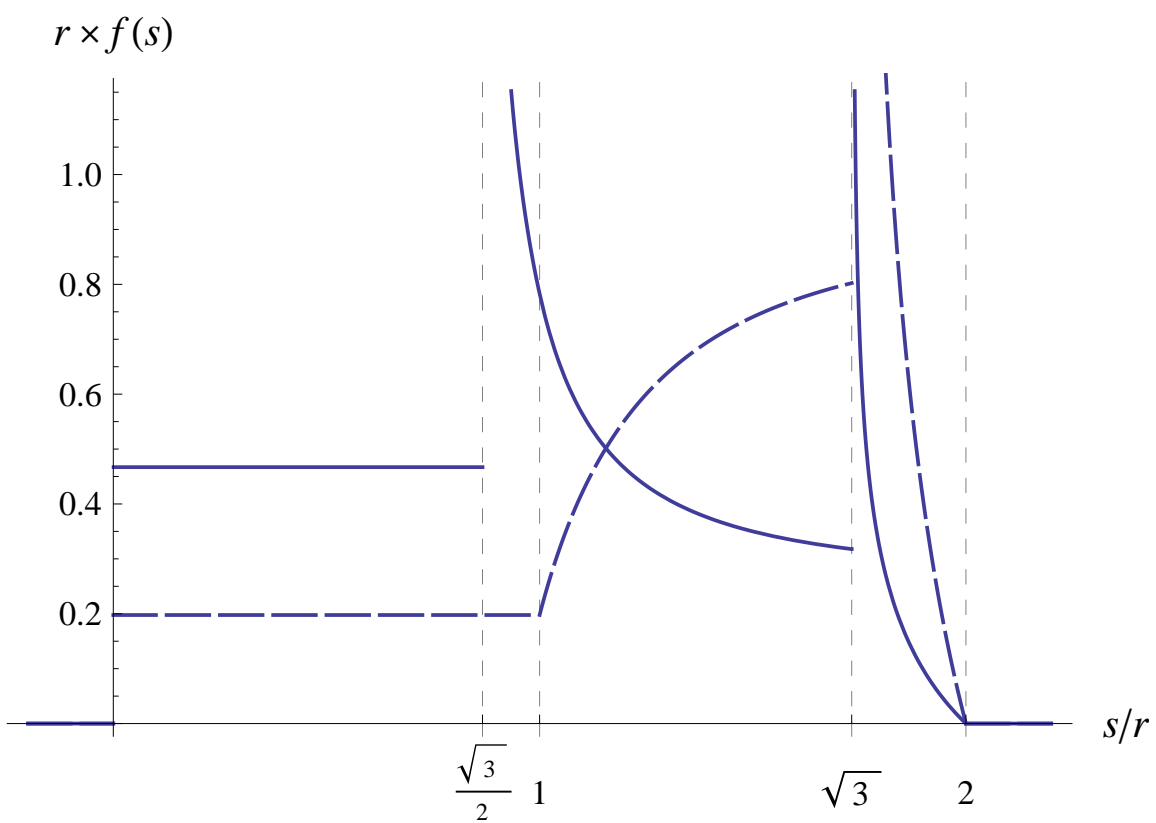

Figure 11. Chord length density function of $\mathcal{H}$ (solid line); chord length density function of the regular hexagon of side length $r$ (dashed line) according to [10] 


\section{The chord length distribution function of a non-convex hexagon}

\section{Conclusions}

The linear part $f_{1}(s)$ of the density function $f(s)$ in Case 1 can also be obtained by Gates' formula [7, p. 866-867]

$$
f_{1}(s)=\frac{1}{2 L}\left[n+\sum_{i=1}^{n}\left(\pi-\gamma_{i}\right) \cot \gamma_{i}\right]
$$

for convex polygons, where $L$ is the perimeter of the polygon and $n$ is the number of its internal angles $\gamma_{i}$. Since $\mathcal{H}$ is non-convex, we only consider the internal angles $\gamma_{i} \leq \pi / 2$. With

$$
\gamma_{1}=\gamma_{2}=\frac{\pi}{3}, \quad \gamma_{3}=\gamma_{4}=\gamma_{5}=\frac{2 \pi}{3}
$$

we get

$$
f_{1}(s)=\frac{1}{12 r}\left[5+\sum_{i=1}^{5}\left(\pi-\gamma_{i}\right) \cot \gamma_{i}\right]=\left(15+\frac{\pi}{\sqrt{3}}\right) \frac{1}{36 r} .
$$

For the expected value $\mathbb{E}[s]$ of the chord length $s$ we find

$$
\begin{aligned}
\mathbb{E}[s] & =\int_{-\infty}^{\infty} s \mathrm{~d} F(s)=\int_{0}^{2 r} s f(s) \mathrm{d} s \\
& =\int_{0}^{h} s f_{1}(s) \mathrm{d} s+\int_{h}^{r} s f_{2}(s) \mathrm{d} s+\int_{r}^{2 h} s f_{3}(s) \mathrm{d} s+\int_{2 h}^{2 r} s f_{4}(s) \mathrm{d} s \\
& =\left(\frac{\pi r}{96 \sqrt{3}}+\frac{5 r}{32}\right)+\left(\frac{29 \pi r}{288 \sqrt{3}}+\frac{r}{96}\right)+\left(\frac{13 \pi r}{72 \sqrt{3}}+\frac{r}{12}\right)+\left(\frac{5 \pi r}{24 \sqrt{3}}-\frac{r}{4}\right) \\
& =\frac{\pi r}{2 \sqrt{3}} .
\end{aligned}
$$

The area $A$ of $\mathcal{H}$ is equal to $\sqrt{3} r^{2}$, and the length $L$ of the boundary $\partial \mathcal{H}$ is equal to $6 r$, hence

$$
\frac{\pi A}{L}=\frac{\sqrt{3} \pi r^{2}}{6 r}=\frac{\sqrt{3} \pi r}{2 \cdot 3}=\frac{\pi r}{2 \sqrt{3}}=\mathbb{E}[s] .
$$

We denote by $I_{n}, n=0,1,2, \ldots$, the $n$-th chord power integral defined by

$$
I_{n}=\int_{G \cap K \neq \emptyset} s^{n} \mathrm{~d} G \quad[15, \text { p. } 46, \text { Eq. (4.3)] }
$$




\section{U. Bäsel, V. Bonanzinga, A. Duma}

In $[15$, p. 47 , Eq. (4.7)] (see also [13, p. 94]) one finds that the expected value $\mathbb{E}[s]$ of a bounded convex set $K$ is given by

$$
\mathbb{E}[s]=\frac{I_{1}}{I_{0}} \quad \text { with } \quad I_{0}=L \quad \text { and } \quad I_{1}=\pi A .
$$

This result for convex sets is also true for the non-convex hexagon $\mathcal{H}$. We note, however, that the measure of all lines intersecting $\mathcal{H}$,

$$
\int_{G \cap \mathcal{H} \neq \emptyset} \mathrm{d} G=2 \int_{0}^{\pi / 2} w(\varphi) \mathrm{d} \varphi=4 r+\sqrt{3} r,
$$

is equal to the perimeter of the convex hull of $\mathcal{H}$, and thus is not equal to the length $L$ of $\partial \mathcal{H}$. In [14, p. 6201-6203] is shown that $\mathbb{E}[s]=\pi A / L$ applies also in general for two planar convex overlapping sets, and, clearly, we may consider $\mathcal{H}$ as the union of two overlapping convex sets.

Our results may be applied in the different fields mentioned in the introduction of the present paper, especially in the small-angle scattering (SAS).

It is possible to use $\mathcal{H}$ in order to disprove conjectures concerning chord length distributions of non convex sets, or check that such a conjecture might be true. Today, there is much more known about distributions for convex sets than for non convex sets.

Furthermore, the results might be used as base for the calculation of the distribution of the distance between two points chosen uniformly at random in the non-convex set $\mathcal{H}$. As already mentioned, for regular polygons this step was done in [2]. Such distributions serve as simple models for the study of wireless ad hoc networks in plane areas (cells).

\section{REFERENCES}

1. Uwe Bäsel, Andrei Duma: Verteilungsfunktionen der Sehnenlänge eines nichtkonvexen Polygons, Fernuniversität Hagen: Seminarberichte aus der Fakultät für Mathematik und Informatik, 84 (2011), 141-151. https://www.fernuni-hagen.de/imperia/md/content/ fakultaetfuermathematikundinformatik/forschung/ berichte_mathematik/berichte_band_84.pdf

2. Uwe Bäsel: Random chords and point distances in regular polygons, Acta Math. Univ. Comenianae, 83, 1 (2014), 1-18. http://www.emis. de/journals/AMUC/_vol-83/_no_1/_baesel/baeselrea.pdf

3. Salvino Ciccariello: The isotropic correlation function of plane figures: the triangle case, Journal of Physics: Conference Series 247 
The chord length distribution function of a non-convex hexagon

(2010) 012014, XIV International Conference on Small-Angle Scattering (SAS09), 1-10. http://iopscience.iop.org/article/10.1088/1742-6596/ $247 / 1 / 012014 / \mathrm{pdf}$

4. Vincenzo Conserva, Andrei Duma: Schnitte eine regulären Hexagons im Buffon- und Laplace-Gitter, Fernuniversität Hagen: Seminarberichte aus der Fakultät für Mathematik und Informatik, 78 (2007), 29-36.

5. Andrei Duma, Sebastiano Rizzo: Chord length distribution functions for an arbitrary triangle, Rend. Circ. Mat. Palermo, Serie II, Suppl. 81 (2009), 141-157. http://math.unipa.it/ circmat/Suppl\%2081\%20PDF. pdf

6. Andrei Duma, Sebastiano Rizzo: La funzione di distribuzione di una corda in un trapezio rettangolo, Rend. Circ. Mat. Palermo, Serie II, Suppl. 83 (2011), 147-160. http://math.unipa.it/ circmat/ Supplemento_83.pdf

7. John Gates: Some properties of chord length distributions, J. Appl. Prob., 24 (1987), 863-874. https://www.jstor.org/stable/3214211

8. Wilfried Gille: Chord length distributions and small-angle scattering, Eur. Phys. J. B, 17 (2000), 371-383. https://link.springer.com/content/ pdf/10.1007\%2Fs100510070116.pdf

9. Wilfried Gille: Particle and Particle Systems Characterization: Small-Angle Scattering (SAS) Applications, CRC Press, Boca Raton/London/New York, 2014.

10. H. S. Harutyunyan: Chord length distribution function for regular hexagon, Uchenie Zapiski, Yerevan State University, 1 (2007), 17-24.

http://www.ysu.am/files/2.CHORD\%20LENGTH\%

20DISTRIBUTION\%20FUNCTION.pdf

11. H. S. Harutyunyan, V. K. Ohanyan: The chord length distribution function for regular polygons, Adv. Appl. Prob. (SGSA), 41 (2009), 358-366. https://www.jstor.org/stable/27793882

12. C. L. Mallows, J. M. C. Clark: Linear-intercept distributions do not characterize plane sets, J. Appl. Prob., 7 (1970), 240-244.

13. Arakaparampil M. Mathai: An Introduction to Geometrical Probability, Gordon and Breach, Australia, 1999.

14. Alain Mazzolo, Benoît Roesslinger, Wilfried Gille: Properties of chord length distributions of nonconvex bodies, Journal of Mathematical Physics, 44, 12 (2003), 6195-6208. http://aip.scitation.org/doi/abs/10. $1063 / 1.1622446$ 


\section{U. Bäsel, V. Bonanzinga, A. Duma}

15. Luis A. Santaló: Integral Geometry and Geometric Probability, AddisonWesley, London, 1976.

16. Loredana Sorrenti: Chord length distribution functions for an isosceles trapezium, General Mathematics, 20, 1 (2012), 9-24. http://depmath. ulbsibiu.ro/genmath/gm/vol20nr1/02_Sorrenti/02_Sorrenti.pdf

17. Rolf Sulanke: Die Verteilung der Sehnenlängen an ebenen und räumlichen Figuren, Math. Nachr., 23 (1961), 51-74. http:// onlinelibrary.wiley.com/doi/10.1002/mana.19610230104/epdf 